\title{
ANÁLISE DA ADESÃo À TERAPIA ANTINEOPLÁSICA ORAL DE \\ PACIENTES ATENDIDOS NA FARMÁCIA DE QUIMIOTERAPIA DE UM \\ HOSPITAL PÚBLICO DE MATO GROSSO DO SUL
}

\author{
ANALYSIS PATIENT ADHERENCE TO THE ANTINEOPLASTIC ORAL \\ THERAPY TREATEMENT IN THE CHEMOTHERAPY PHARMACY AT \\ HOSPITAL OF MATO GROSSO DO SUL
}

\section{Recebimento:30/11/2017- Aceite: 09/01/2018- Publicação: 28/01/2018 \\ Processo de Avaliação: Double Blind Review}

\author{
Fabiana Mesquita Roese \\ Mestrado em Saúde e Desenvolvimento na Região Centro-Oeste \\ Universidade Federal de Mato Grosso do Sul \\ fabi.roese@gmail.com

\section{Eliane Moro Fontana} \\ Especialista em Manipulação de Medicamentos e Cosméticos \\ Conselho Regional de Farmácia do Mato Grosso do Sul \\ elianefont@hotmail.com
}

Karen Carla de Brito Pereira

Graduada em Farmácia

Universidade para o Desenvolvimento do Estado e da Região do Pantanal

karencarla01@gmail.com

\section{RESUMO}

O câncer de mama é a maior causa de morte por neoplasia no gênero feminino. A terapia hormonal adjuvante é um importante aliado no tratamento do câncer de mama porque melhora a sobrevida e diminui a recorrência da neoplasia. O Tamoxifeno tem sido amplamente utilizado em pacientes com neoplasia de mama que apresentam receptor de estrógeno (RE) positivo por um prazo de até 5 anos. A aceitação do paciente ao tratamento é um importante componente da atenção farmacêutica. Esta pesquisa tem por objetivo analisar a adesão à terapia antineoplásica oral de pacientes atendidos na farmácia de quimioterapia do Hospital Regional de Mato Grosso do Sul. Trata-se de um estudo observacional descritivo retrospectivo de julho a dezembro de 2016, realizado com pacientes maiores de 18 anos ( $\mathrm{n}=95)$ em uso de hormonioterapia com Tamoxifeno. Observou-se que a maioria dos pacientes estava na faixa etária de 42-60 anos, solteiros, brancos e procedentes de Campo Grande. No estudo, 30,5\% dos pacientes deixaram de retirar a medicação em algum dos meses avaliados. A participação do farmacêutico

Revista ENIAC Pesquisa, Guarulhos (SP), V.7, n.1, jan.- jun. 2018. 
junto à equipe multidisciplinar e ao paciente é essencial no manejo dos efeitos colaterais, reações adversas e promoção da adesão.

Palavras-chave: Adesão à medicação; Modulador Seletivo dos Receptores de Estrógeno; Neoplasia da Mama; Atenção Farmacêutica.

\begin{abstract}
Breast cancer is the leading cause of death due to neoplasm in the female gender. Adjuvant hormone therapy is an important ally in breast cancer treatment because it improves survival and decreases the recurrence of neoplasm. Tamoxifen has been widely used in patients with breast cancer who present positive estrogen receptor (ER) for up to 5 years. Patient treatment acceptance is an important component of pharmaceutical care. This study aims to analyze patient adherence to the antineoplastic oral therapy treatment in the chemotherapy pharmacy at Regional Hospital of Mato Grosso do Sul. This is a retrospective descriptive observational study from July to December 2016, conducted with patients older than 18 years $(n=95)$ using hormone therapy with Tamoxifen. Results: It was observed that most of the patients were in the age range of 42-60 years, single, white and coming from Campo Grande. In the study, $30.5 \%$ of the patients stopped withdrawing the medication in any of the evaluated months. The participation of the pharmacist jointly the multidisciplinary team and the patient is essential in managing side effects, adverse reactions and promotion of adherence.
\end{abstract}

Keywords: Medication Adherence; Selective Estrogen Receptor Modulators Therapy; Breast Neoplasm; Pharmaceutical Care.

\title{
1. INTRODUÇÃO
}

Todos os anos mais de 14 milhões de pessoas são diagnosticadas com alguma neoplasia no mundo. Em 2015 foram 8,8 milhões de mortes, evidenciando que uma em cada seis mortes no mundo foi por câncer. Pode-se concluir que o câncer mata mais do que HIV/AIDS, tuberculose e malária juntos (WHO, 2017).

Entre as mulheres, o câncer de mama tem uma alta incidência com uma taxa de 43,3 para cada 100. 000 mulheres. Apesar desse número elevado há uma baixa taxa de mortalidade equivalente a 12,9 para cada 100.000 mulheres, porém ainda é a maior causa de morte por câncer no gênero feminino (STEWART e WILD, 2014).

O Instituto Nacional de Câncer (INCA) estimou para no ano de 2016, 57.900 novos casos de câncer de mama no Brasil, com um risco estimado de 56,20 casos a cada 100 mil mulheres. É considerado o mais frequente nas mulheres das regiões Sul,

Revista ENIAC Pesquisa, Guarulhos (SP), V.7, n.1, jan.- jun. 2018. 
Sudeste, Centro-Oeste e Nordeste, desconsiderando os tumores de pele não melanoma (INCA, 2011).

Os sintomas mais perceptíveis do câncer de mama são: pele avermelhada e com aspecto de casca de laranja, alterações no mamilo, saída de secreção da mama e nódulos no pescoço ou axilas (INCA, 2017).

O câncer de mama tem bom prognóstico se realizado tratamento precoce, no entanto não tem objetivo curativo quando se torna metastático. A sobrevida no Brasil após 5 anos do diagnóstico é de $80 \%$, considerando que em países desenvolvidos chega a $85 \%$. Porém se for considerado metastático a média é de 2 anos, podendo variar de meses a anos (BRASIL, 2015).

As terapias antineoplásicas por via oral estão se tornando cada vez mais presentes na vida dos pacientes oncológicos. Porém demanda maior autonomia e exige maior responsabilidade por parte dos pacientes na gestão do tratamento (TOKDEMIR e KAV, 2017).

A terapia hormonal com antiestrogênios constitui uma peça fundamental do tratamento endócrino do câncer de mama com receptores hormonais positivos. Dentre as terapias orais atuais para a neoplasia de mama estão: o Tamoxifeno, o Anastrozol, o Letrozol e Exemestano (CHABNER et al., 2015).

O Tamoxifeno pertence ao grupo dos moduladores seletivos dos receptores de estrogênio (SERM). É utilizado clinicamente em mulheres com câncer de mama com receptores positivos para estradiol (RE), como adjuvante pós-operatório, administrado por um período de até 5 anos. Reduz a probabilidade anual de recidiva de câncer de mama em $39 \%$ e a probabilidade anual de morte em $31 \%$, com efeitos semelhantes independentemente da idade, do estado de menopausa e dos linfonodos (CHABNER et al., 2015)

Anastrozol, Letrozol e Exemestano são Inibidores da Aromatase (IAs) são utilizados no tratamento do câncer de mama metastáticos com (RE) positivos e como adjuvante no tratamento do câncer de mama em estágio inicial. Apresentam superioridade em relação ao Tamoxifeno no tratamento das mulheres pós-menopausa (CHABNER et al., 2015).

A adesão do paciente ao tratamento é um importante componente da atenção farmacêutica. A falta de aderência à terapia é um problema mundial, a taxa em países

Revista ENIAC Pesquisa, Guarulhos (SP), V.7, n.1, jan.- jun. 2018. 
desenvolvidos chega a 50\%. Em países em desenvolvimento esse número é ainda menor, demonstrando que os pacientes apresentam dificuldades na continuidade da terapia proposta. $\mathrm{O}$ impacto cresce proporcionalmente as doenças crônicas, compromete a eficácia do tratamento, resultando em aumento dos custos com saúde (STEWART e WILD, 2014).

Considerando as informações expostas, tem-se por objetivo analisar o perfil de adesão de pacientes de hormonioterapia com o medicamento Tamoxifeno atendidos pela farmácia de quimioterapia de um hospital público de Mato Grosso do Sul no período de julho a dezembro de 2016 e sugerir ferramentas que favoreçam a adesão ao tratamento.

\section{METODOLOGIA}

Trata-se de um estudo epidemiológico retrospectivo descritivo, realizado no HRMS (Hospital Regional de Mato Grosso do Sul) nos meses de julho a dezembro de 2016.

O Hospital Regional de Mato Grosso de Sul foi inaugurado em 1997, contemplando 352 leitos e conta com 45 especialidades médicas divididas em sete linhas de cuidados: Cardiovascular, Clínica Médica, Materno-Infantil, Nefro-Urológica, Paciente Crítico e Oncológica (Oncologia Clínica e Hematologia).

Foi solicitada autorização para a realização de pesquisa ao Comitê de Ética em Pesquisa do Hospital Regional de Mato Grosso do Sul, conforme preconiza a resolução CNS 466 de 12 de dezembro de 2012 e demais resoluções complementares. Após a aprovação foi submetido ao Comitê de Ética em Pesquisa com Seres Humanos da universidade Anhanguera - UNIDERP e aprovado sob o protocolo de $\mathrm{n}^{\circ}$ 68987717.80000 .5161 .

Os dados analisados foram provenientes dos prontuários médicos e dos relatórios de dispensação dos pacientes acompanhados pelo setor de Oncologia com o diagnóstico de câncer de mama e somente daqueles tratados com Tamoxifeno que fizeram a retirada do medicamento na Farmácia de Quimioterapia. Foi solicitado dispensa do Termo de Consentimento Livre e Esclarecido tendo em vista que foram utilizados somente dados secundários obtidos a partir do estudo de material já coletado para fins de revisão.

Revista ENIAC Pesquisa, Guarulhos (SP), V.7, n.1, jan.- jun. 2018. 
População: pacientes do gênero feminino maiores de 18 anos com diagnóstico de câncer de mama que realizaram tratamento com Tamoxifeno dipensado pela Farmácia de Quimioterapia do HRMS.

Amostra: a amostra foi por conveniência e analisados os prontuários nas variáveis: idade, gênero, estado civil, cor, cidade de origem, diagnóstico.

Critérios de inclusão: prescrição de medicamento dispensado pela Farmácia de Quimioterapia (Tamoxifeno) de pacientes hospitalizados e ambulatoriais dos quais o prontuário continha a informação de que o paciente estava em uso do Tamoxifeno.

Critérios de Exclusão: prescrição pediátrica, prescrição de medicamentos administrados por via endovenosa, subcutânea e intratecal. Outros medicamentos de via oral que não o Tamoxifeno.

As informações de idade, gênero, cor, cidade de origem e estado civil foram retiradas do prontuário eletrônico PEP do SOUL MV®, através do número do prontuário fornecido nas prescrições enviadas para a Farmácia de Quimioterapia realizar as dispensações.

Essas informações foram armazenadas no programa Excell utilizando apenas número para identificação dos pacientes. Para tabulação dos dados foi utilizado o software Microsoft Office Excell 2010 e a análise foi realizada com os programas GraphPad Prism 5 e Epi Info 7.

\section{ANÁLISE DOS DADOS}

Foram incluídos no estudo 95 pacientes do gênero feminino e masculino. Houve apenas um paciente do gênero masculino, que inclusive não retirou a medicação em nenhum dos meses analisados. É portador de Alzheimer e dependente do acompanhante. Um paciente do gênero feminino foi excluído do estudo, pois não foi possível acessar o prontuário eletrônico. Dois pacientes deixaram de retirar a medicação. Um apresentou recidiva e outro evolui com metástase, havendo necessidade de mudança no protocolo utilizado.

Durante o período de análise a média mensal de atendimentos realizados pela Farmácia de Quimioterapia do Hospital Regional de Mato Grosso do Sul foi de 461

Revista ENIAC Pesquisa, Guarulhos (SP), V.7, n.1, jan.- jun. 2018. 
(figura 1); julho (424), agosto (460), setembro (474), outubro (448), novembro (492) e dezembro (468).

Considerando que um paciente pode ter sido atendido mais de uma vez no período analisado. Nota-se que houve um aumento de julho a novembro, sendo este último o mês com maior atendimento e um decréscimo em dezembro. Na figura 1, pode-se observar também que os atendimentos correspondem a quimioterápicos manipulados e pela dispensação de comprimidos para tratamento domiciliar.

Evidencia também o número de pacientes novos atendidos: julho (18), agosto (35), setembro (40), outubro (32), novembro (31) e dezembro (24). Houve um aumento progressivo de julho a setembro e uma queda em de outubro a dezembro, divergindo da média de atendimentos. O mês de setembro foi o que mais apresentou casos novos.

O Hospital Regional atendeu uma média de 154 pacientes com neoplasia de mama que correspondeu a $33,40 \%$ da média total de atendimentos de julho a dezembro de 2016. Dos 154 pacientes, uma média de 89,33 estava em hormonioterapia com Tamoxifeno.

As informações coletadas mostraram a média mensal de 802,5 doses de manipulação de quimioterapia para pacientes adultos da oncologia clínica e hematologia. A média mensal de dispensação para uso domiciliar nesse mesmo período foi de 431,8 .

A Figura 1, apresenta o número total de atendimentos e de pacientes novos atendidos pela Farmácia de Quimioterapia do Hospital Regional de Mato Grosso do Sul no período de julho a dezembro de 2016.

Revista ENIAC Pesquisa, Guarulhos (SP), V.7, n.1, jan.- jun. 2018. 
Figura 1: Número total de atendimentos no período de julho a dezembro de 2016

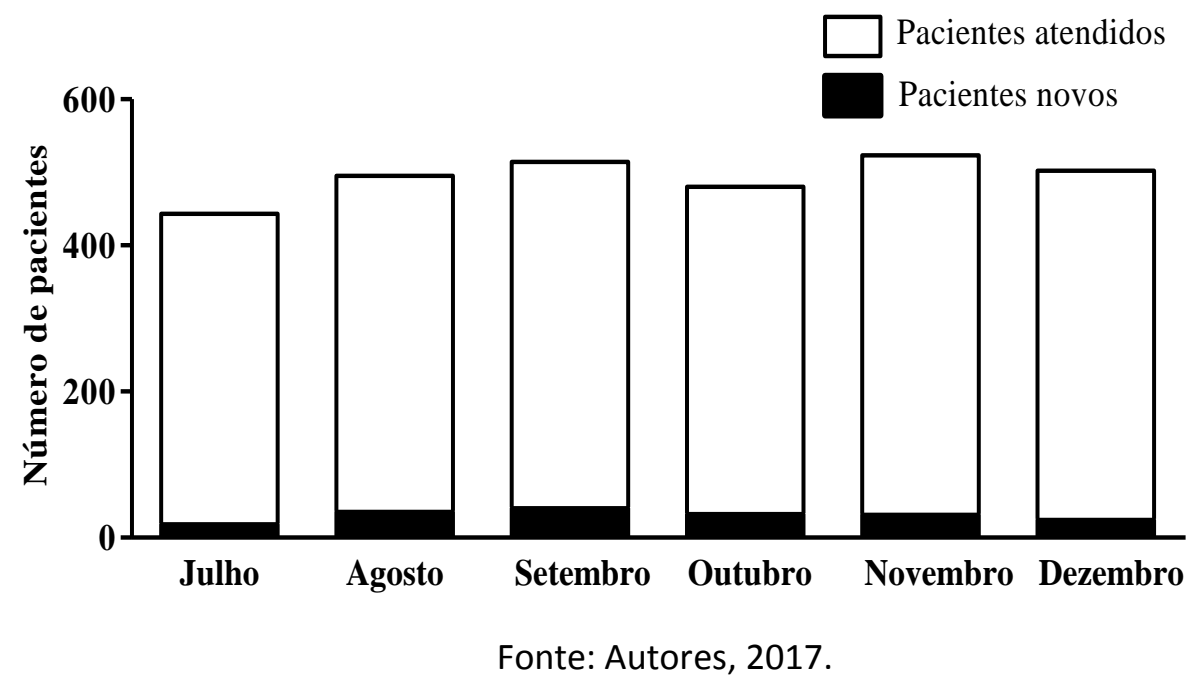

Os dados apresentados na tabela 1 destacam que o mês de julho foi o que mais apresentou adesão, enquanto que o mês de novembro foi o que revelou uma menor porcentagem de dispensação do medicamento. Informação que pode estar associada ao fato desses meses coincidirem com datas festivas de final de ano e férias.

A Tabela 1, apresenta o número e porcentagem de pacientes com câncer de mama que aderiram ou não ao tratamento com o medicamento de via oral Tamoxifeno no Hospital Regional de Mato Grosso do Sul no período de julho a dezembro de 2016.

Tabela 1: Porcentagem de pacientes com câncer de mama no período de julho a dezembro de 2016.

\begin{tabular}{|c|c|c|c|c|c|c|}
\hline \multirow{3}{*}{ Variável } & \multicolumn{4}{|c|}{ Adesão ao Tamoxifeno } & & \\
\hline & \multicolumn{2}{|c|}{ Sim } & \multicolumn{2}{|c|}{ Não } & \multicolumn{2}{|c|}{ Total } \\
\hline & $\mathbf{N}^{\circ}$ & $\%$ & $\mathbf{N}^{\circ}$ & $\%$ & $\mathbf{N}^{\circ}$ & $\%$ \\
\hline \multicolumn{7}{|l|}{ Meses } \\
\hline Julho & 75 & 90,4 & 8 & 9,6 & 83 & 100 \\
\hline Agosto & 73 & 83,9 & 14 & 16,1 & 87 & 100 \\
\hline Setembro & 74 & 83,1 & 15 & 16,9 & 89 & 100 \\
\hline Outubro & 74 & 82,2 & 16 & 17,8 & 90 & 100 \\
\hline Novembro & 75 & 81,5 & 17 & 18,5 & 92 & 100 \\
\hline Dezembro & 81 & 85,3 & 14 & 14,7 & 95 & 100 \\
\hline
\end{tabular}

* Um mesmo paciente pode ter sido atendido por mais de um mês, com média de atendimento mensal de 89 pacientes

Fonte: Autores, 2017.

Revista ENIAC Pesquisa, Guarulhos (SP), V.7, n.1, jan.- jun. 2018. 
A Figura 2, apresenta o número de pacientes com câncer de mama que aderiram de forma ininterrupta ao tratamento com o medicamento de via oral Tamoxifeno no Hospital Regional de Mato Grosso do Sul no período de julho a dezembro de 2016 ( $\mathrm{n}=$ 95).

Figura 2 pacientes com câncer de mama que aderiram de forma ininterrupta ao tratamento com o medicamento de via oral

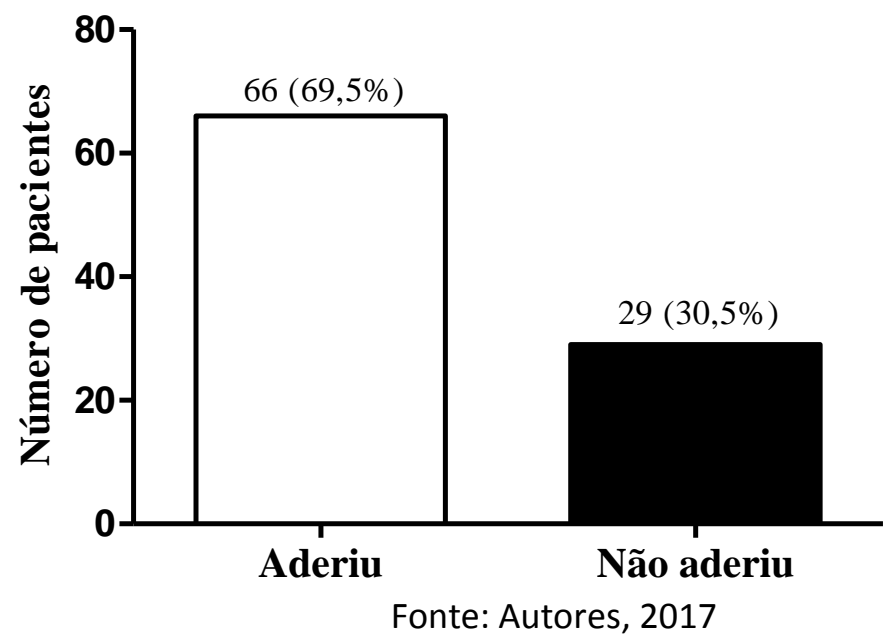

Considerando-se a adesão ao tratamento com Tamoxifeno de julho a dezembro de 2016 de forma ininterrupta, o número total de pacientes foi de 66 (69,5\%) (figura 2). Enquanto que o número de pacientes que deixaram de retirar o medicamento em algum dos meses foi de $29(30,5 \%)$.

Durante o estudo de Veloso e colaboradores (2012), empregando o teste de Morisky e Green para mulheres em uso de Tamoxifeno, foi observado que 52,1\% dos pacientes foram aderentes. Cabe ressaltar, que o teste avalia os pacientes com questionário, portanto é mais fidedigno para comprovar adesão e não adesão. A presente investigação avaliou apenas se o paciente compareceu ou enviou representante para retirar sua medicação nos meses propostos, por essa razão a adesão pode ter se mostrado maior. No mesmo estudo observou-se que 64\% dos pacientes em uso de terapia antineoplásica oral com Tamoxifeno, Anastrozol, Megestrol e Etoposídeo, foram não aderentes pelo menos uma vez nos meses avaliados. O Tamoxifeno e o Anastrozol foram os que tiveram maior descontinuidade de tratamento.

Revista ENIAC Pesquisa, Guarulhos (SP), V.7, n.1, jan.- jun. 2018. 
De acordo com Oliveira (2012) alguns fatores influenciam a adesão, porém $73,6 \%$ dos pacientes abordados discordaram quanto aos efeitos indesejáveis ser um fator para não adesão ao tratamento com Tamoxifeno. $\mathrm{O}$ acesso ao medicamento também não foi exposto como problema $(98,1 \%)$, pois eles estavam disponíveis na farmácia ambulatorial. $\mathrm{O}$ esquecimento foi referido por $32,1 \%$ dos pacientes.

É importante destacar que a atenção farmacêutica ao paciente que faz uso da terapia oral pode contribuir positivamente para adesão ao tratamento, pois nesse processo é possível detectar falhas, tirar dúvidas, solucionar problemas relacionados ao uso de medicamentos e atender o paciente de forma individualizada de acordo com suas necessidades (VELOSO et al., 2012).

Tabela 2: Características dos pacientes com câncer de mama atendidos no período de julho a dezembro de 2016

\begin{tabular}{lrr}
\hline \multicolumn{1}{c}{ Variável } & Número de pacientes & \% de pacientes \\
\hline Idade & 2 & \\
20 a 30 anos & 9 & $2,1 \%$ \\
31 a 40 anos & 27 & $9,5 \%$ \\
41 a 50 anos & 20 & $28,4 \%$ \\
51 a 60 anos & 20 & $21,1 \%$ \\
61 a 70 anos & 16 & $21,1 \%$ \\
71 a 80 anos & 1 & $16,8 \%$ \\
81 a 90 anos & & $1,0 \%$ \\
Estado civil & 76 & \\
Solteiro (a) & 15 & $80,0 \%$ \\
Casado (a) & 2 & $15,8 \%$ \\
Divorciado (a) & 2 & $2,1 \%$ \\
Viúvo (a) & & $2,1 \%$ \\
Grupo étnico & 37 & $39,0 \%$ \\
Branco & 5 & $5,3 \%$ \\
Preto & 18 & $18,9 \%$ \\
Pardo & 35 & $36,8 \%$ \\
Ignorado & & \\
Cidade onde mora & & $69,5 \%$ \\
Campo Grande & 66 & $3,2 \%$ \\
Coxim & 3 & $4,1 \%$ \\
Ribas do Rio Pardo & 4 & $3,2 \%$ \\
Rio Brilhante & 3 & $20,0 \%$ \\
Outras & 19 & $100 \%$ \\
\hline Total & 95 & \\
\hline
\end{tabular}

Fonte: Autores, 2017.

Revista ENIAC Pesquisa, Guarulhos (SP), V.7, n.1, jan.- jun. 2018. 
A Tabela 2, apresenta a análise das características dos pacientes com câncer de mama atendidos no período de julho a dezembro de 2016 no Hospital Regional de Mato Grosso do Sul (n=95).

A faixa etária prevalente nos atendimentos da dispensação oral foi de 41-50 anos $(28,4 \%)$, seguido pelas faixas de 51-60 (21,1\%) e 61-70 (21,1\%), (Tabela 2). A faixa com menor representatividade foi de 81-90 anos. As informações corroboram com os dados obtidos pelo estudo realizado com mulheres em tratamento de câncer de mama com Tamoxifeno do Hospital Santa Rita de Cássia (HSRC), que aponta que a maioria das mulheres, cerca de 66\% está na faixa etária de 41-60 anos (LEITE, 2011).

Quanto ao estado civil, $80 \%$ dos pacientes declararam ser solteiros, e apenas $15,8 \%$ casados. O que diverge do estudo de Leite e colaboradores (2011), em que a maioria se declarou casado (64\%) ou com companheiro. Essa diferença pode estar associada ao fato das informações terem sido retiradas dos prontuários e dos pacientes não serem abordados sobre a situação marital não oficializada. Os dados obtidos também diferem da análise realizada no sul do Brasil onde $64,3 \%$ dos pacientes eram casados (RÊGO e NERY 2013).

Em relação ao grupo étnico, 39\% dos pacientes se declararam brancos, seguido do pardo (18,9\%). Destaca-se também que em 36,8\% dos prontuários a informação sobre etnia foi ignorada. Os dados obtidos discordaram do estudo realizado em mulheres com câncer de mama no Piauí, onde o grupo pardo foi prevalente (48\%). Já em estudo realizado por Dugno (2014) na região Sul a população do estudo apresentou predominância branca $(92,7 \%)$. Essa diferença pode estar associada ao padrão sociodemográfico do Mato Grosso do Sul e a dificuldade em se classificar adequadamente os pacientes quanto a essa característica.

A cidade de Campo Grande foi a que obteve maior porcentagem como local de origem dos pacientes $(69,5 \%)$, divergindo do estudo realizado em um hospital oncológico de Teresina que avaliou 50 mulheres com câncer de mama em que a maioria dos pacientes era do interior (62,00\%) (RÊGO e NERY, 2013).

A Tabela 3, apresenta a correlação entre a adesão ao tratamento com o medicamento de via oral Tamoxifeno e as variáveis idade, estado civil, grupo étnico e cidade dos pacientes com câncer de mama atendidos no Hospital Regional de Mato Grosso do Sul no período de julho a dezembro de 2016 ( $\mathrm{n}=95)$.

Revista ENIAC Pesquisa, Guarulhos (SP), V.7, n.1, jan.- jun. 2018. 
Tabela 3: Correlação entre a adesão ao tratamento com o medicamento de via oral Tamoxifeno.

\begin{tabular}{|c|c|c|c|c|c|c|}
\hline \multirow{3}{*}{ Variáveis } & \multicolumn{4}{|c|}{ Adesão } & & \\
\hline & \multicolumn{2}{|c|}{ Sim } & \multicolumn{2}{|c|}{ Não } & \multicolumn{2}{|c|}{ Total } \\
\hline & $\mathbf{N}^{\circ}$ & $\%$ & $\mathbf{N}^{\circ}$ & $\%$ & $\mathbf{N}^{\circ}$ & $\%$ \\
\hline \multicolumn{7}{|l|}{ Idade } \\
\hline 20 a 40 anos & 5 & 54,5 & 6 & 45,5 & 11 & 100 \\
\hline 41 a 60 anos & 37 & 78,7 & 10 & 21,3 & 47 & 100 \\
\hline 61 a 90 anos & 24 & 64,9 & 13 & 35,1 & 37 & 100 \\
\hline \multicolumn{7}{|l|}{ Estado civil } \\
\hline Solteiro (a) & 53 & 69,7 & 23 & 30,3 & 76 & 100 \\
\hline Casado (a) & 11 & 73,3 & 4 & 26,7 & 15 & 100 \\
\hline Divorciado (a) & 1 & 50,0 & 1 & 50,0 & 2 & 100 \\
\hline Viúvo (a) & 1 & 50,0 & 1 & 50,0 & 2 & 100 \\
\hline \multicolumn{7}{|l|}{ Grupo étnico } \\
\hline Branco & 25 & 67,6 & 12 & 32,4 & 37 & 100 \\
\hline Preto & 3 & 60,0 & 2 & 40,0 & 5 & 100 \\
\hline Pardo & 14 & 77,8 & 4 & 22,2 & 18 & 100 \\
\hline Ignorado & 24 & 68,6 & 11 & 31,4 & 35 & 100 \\
\hline \multicolumn{7}{|l|}{ Cidade } \\
\hline Campo Grande & 46 & 69,7 & 20 & 30,3 & 66 & 100 \\
\hline Interior & 20 & 69,0 & 9 & 31,0 & 29 & 100 \\
\hline
\end{tabular}

O grupo para o qual a farmácia de quimioterapia do HRMS mais dispensou Tamoxifeno foi para a faixa etária de 41-60 anos (78,7\%). Segundo o estado civil, os pacientes casados contemplaram $73,3 \%$ e os solteiros $69,7 \%$. O grupo étnico mais representativo na adesão ao tratamento foi o branco $(n=25)$, seguido pelo pardo $(n=14)$. Os prontuários onde a informação de etnia foi ignorada apresentaram-se expressivos $(68,6 \%)$. O fato de ser de Campo Grande ou do interior do Estado não influenciou, embora os pacientes provenientes da capital sejam um número maior (Tabela 3).

Em relação aos não aderentes, a faixa etária de 20 a 40 anos representou 45,5\% dos pacientes, em segundo lugar a faixa de 61 a 90 anos com 35,1\%, apesar de ter um número total de pacientes $(n=13)$ maior do que o grupo de 20 a $40(n=6)$.

A não aderência das pacientes pode estar associada aos efeitos colaterais do medicamento. Na análise de Rêgo e Nery (2013) que entrevistou mulheres em uso de Tamoxifeno, observou-se que os efeitos colaterais estiveram presentes em $87 \%$ dos pacientes entrevistados, incluindo efeitos vasomotores (fogachos), ginecológicos, entre outros.

Revista ENIAC Pesquisa, Guarulhos (SP), V.7, n.1, jan.- jun. 2018. 
Durante estudo com dados secundários de pacientes em hormonioterapia no INCA estimou-se que o risco de descontinuidade do tratamento em mulheres com idade inferior a 40 anos é $25 \%$ maior quando comparado ao restante do grupo (BRITO, PORTELA, VASCONCELLOS, 2014).

A idade sexualmente ativa das mulheres também influencia, pois o medicamento apresenta como efeito colateral a diminuição da libido. Castro (2016) afirma que o impacto do câncer de mama é maior para pessoas jovens e menos negativo para mulheres mais velhas.

Os divorciados e viúvos representaram cada um 50\% de não aderência, porém o número não é expressivo, pois nessa análise obteve um representante cada. Portanto, deve-se levar em consideração como mais prevalente para não aderência o grupo declarado solteiro com 30,3\%. Uma revisão sistemática aponta que o fato do indivíduo não ser casado aumenta o risco de apresentar câncer em estágio avançado. Enquanto que estar em um relacionamento apresentou estatística significativa para detecção precoce do câncer de mama (BUJA et al., 2017; LANNIN, 1998).

O grupo étnico preto foi pouco aderente quando comparado aos outros, porém o número de pacientes foi menor $(n=2)$. Logo após vem o grupo branco $(n=12)$. Os dados ignorados para grupo étnico também foram significativos (31,4\%). Rêgo e Nery (2013) também constataram que $100 \%$ dos pacientes do grupo étnico preto $(\mathrm{n}=8)$, apresentaram dificuldade quanto a cumprir as etapas do tratamento do câncer de mama em discordância com os $88,9 \%$ do grupo branco $(n=8)$.

A localização da residência não influenciou a retirada do medicamento, informação semelhante foi demonstrada por pesquisadores que avaliaram o perfil dos pacientes atendidos na Farmácia Ambulatorial do Hospital de Câncer II, que aponta que não existe correlação entre o local da residência e a dificuldade de retirada dos medicamentos, mas entre o tempo de tratamento e uso dos medicamentos foi possível identificar uma relação (VELOSO et al., 2012).

No grupo dos pacientes não aderentes, as queixas que foram possíveis de associar ao uso de Tamoxifeno mediante a avaliação do prontuário foram: hiporexia, algias, cefaleias, nervosismo, manchas na pele e aumento de peso. Como descrito na literatura em média 30\% dos pacientes apresentam: sintomas vasomotores (fogachos), secreção vaginal, retenção hídrica e perda da libido como efeitos colaterais mais

Revista ENIAC Pesquisa, Guarulhos (SP), V.7, n.1, jan.- jun. 2018. 
comuns. Em torno de $10 \%$ a 30\% dos casos relatam náuseas, irregularidades menstruais, sangramento vaginal e alterações de humor (CHABNER et al., 2015).

Apesar de não haver relatos dos sintomas vasomotores neste estudo, mulheres em pré-menopausa que fazem tratamento com hormonioterapia podem apresentar esse sintoma em 95\% dos casos (HICKEY et al.,2005). Em avaliação do estado nutricional de mulheres em uso de Tamoxifeno da Associação de Voluntários no Combate ao Câncer (AVACCI) em Itauna-MG, 52,4 \% estavam em sobrepeso e 45,8\% com obesidade (LAGARES, 2013).

A depressão atinge cerca de $10 \%$ dos pacientes com neoplasia de mama (SOUZA et al., 2014). Significa que muitos deles fazem uso de antidepressivos como os Inibidores Seletivos da recaptação da Serotonina (ISRS), não só para depressão, como também para tratamento das ondas de calor (fogachos) (FÉLIX, AOKI e LIMA, 2010).

No entanto, os antidepressivos devem ser prescritos com cautela, pois as interações medicamentosas entre o Tamoxifeno e os ISRS levam a diminuição da ação da principal enzima responsável pela metabolização Endoxifeno (CYP2D6), restringindo assim, a formação desse composto (CHABNER et al., 2015).

Para que o pró-fármaco Tamoxifeno seja convertido em seu metabólito ativo o Endoxifeno, o sistema Citocromo P450 precisa estar atuante (CHABNER et al., 2015).

Essa interação interfere na resposta clínica e gera maior susceptibilidade de recorrência da neoplasia (BINKHORST et al., 2013). Alguns dos prontuários analisados demonstravam o uso concomitante dessas medicações, inclusive porque um representante dos ISRS é a fluoxetina, medicamento padronizado pela Atenção Básica.

Não foi possível demonstrar uma significância estatística sobre a não adesão e a presença de efeitos colaterais, a maioria dos prontuários não continha essa associação. Com base nessa constatação uma análise empregando o questionário de Morisky e Green poderia elucidar com maior clareza esse questionamento.

O perfil socioeconômico e a escolaridade também são fatores que podem influenciar o grau de adesão, demonstrando que essas informações precisam ser estudadas.

Revista ENIAC Pesquisa, Guarulhos (SP), V.7, n.1, jan.- jun. 2018. 


\section{CONCLUSÕES}

O câncer de mama é uma questão de saúde pública, afeta na sua maioria mulheres jovens e produtivas como demonstrado neste estudo (41-50) anos. O fato de $30,5 \%$ dos pacientes deixarem de utilizar o medicamento proposto pelo menos uma vez no período do estudo é preocupante, pois a falha terapêutica é iminente.

Questões como escolaridade devem ser mais bem analisadas, pois é possível que muitos dos pacientes não aderentes, não tenham compreendido durante a consulta médica a real importância do tratamento.

A localização da residência do paciente não influenciou, mas talvez a longa jornada do tratamento possa gerar desgastes no paciente, levando a não aderência a terapia oral.

A participação do farmacêutico junto à equipe multidisciplinar e ao paciente é essencial no manejo dos efeitos colaterais e reações adversas. É o profissional que pode melhorar a qualidade de vida dos pacientes, intervindo e contribuindo com a resposta clínica.

O farmacêutico pode utilizar estratégias para fortalecer os vínculos com os pacientes. Uma dessas ferramentas é a Roda de Conversa que tem por finalidade identificar as necessidades, discutir os problemas e encontrar soluções para resolução dos conflitos relacionados ao uso de medicamentos. $\mathrm{O}$ atendimento individual também é um recurso que pode ser empregado para o paciente que necessita de um acompanhamento mais específico.

\section{REFERÊNCIAS}

BINKHORST L, MATHIJSSEN J.H.R, VAN HERK-SUKEL MPP et al. Unjustified prescribing of CYP2D6 inhibiting SSRIs in women treated with tamoxifen.Breast cancer res treat, 2013, 139:923-929.

BRASIL. Lei $\mathrm{n}^{\circ} 11.664$, de 29 de abril de 2.008. Dispõe sobre a efetivação de ações de saúde que assegurem a prevenção, a detecção, o tratamento e o seguimento dos cânceres do colo uterino e de mama, no âmbito do Sistema Único de Saúde - SUS. Brasília, 2008.

Revista ENIAC Pesquisa, Guarulhos (SP), V.7, n.1, jan.- jun. 2018. 
BRASIL. AGÊNCIA NACIONAL DE SAÚDE SUPLEMENTAR (ANS). Resolução Normativa $\mathrm{RN} \mathrm{N}^{\circ} 349$ de 9 de maio de 2014. Regulamenta o tratamento antineoplásico de uso oral. Brasília, 2014.

BRASIL. CONSELHO NACIONAL DE SECRETÁRIOS DE SAÚDE. Assistência Farmacêutica no SUS / Conselho Nacional de Secretários de Saúde. - Brasília: CONASS, 2007.

BRASIL. MINISTÉRIO DA SAÚDE (BR). Instituto Nacional do Câncer. Falando sobre câncer de mama. Rio de Janeiro: INCA; 2002.

Portaria $\mathrm{n}^{\mathrm{o}} 1.008$, de 30 de setembro de 2015. Ministério da Saúde. Secretaria de Atenção à Saúde. Diretrizes Diagnósticas e Terapêuticas do Carcinoma de Mama. Brasília, 2015.

. Portaria $n^{\circ} 3.916$, DE 30 DE OUTUBRO DE 1998. Aprova a Política Nacional de Medicamentos. Brasília, 1998.

Resolução n ${ }^{\circ}$ 338, DE 06 DE MAIO DE 2004. Aprova a Política Nacional de Assistência Farmacêutica. Brasília, 2004.

Secretaria de Vigilância em Saúde Programa Nacional de DST e AIDS. Diretrizes para o fortalecimento das ações de adesão ao tratamento para pessoas que vivem com HIV e AIDS, 2007. Brasília, 2007.

BRITO, C.; PORTELA, M.C. VASCONCELLOS, M.T.L. Fatores associados à persistência à terapiahormonal em mulheres comcâncer de mama. Rev. Saúde Pública 2014;48(2):284-295.

BUJA A, LAGO L, LAGO S, VINELLI A, ZANARDO C, BALDO V. Marital status and stage of cancer at diagnosis: A systematic review. European journal of cancer care, 2017.

CASTRO, E.K.K., LAWRENZ, P.; ROMEIRO, F.; LIMA, N.T. Percepção da doença e enfrentamento em mulheres com câncer de mama. Psicologia: Teoria e Pesquisa Jul-Set 2016, Vol. 32 n. 3, pp. 1-6

CAVA, P. K. P. Importância da adesão ao tratamento com quimioterápicos de uso oral: contribuições do farmacêutico SOBRAFONews, Maio - Junho , 2016.

CHABNER, B. A. et al. Manual de Oncologia de Harrison. $2^{\circ}$ edição. Porto Alegre: AMGH, 2015. Pag.133.

DUGNO, M.L.G.; SOLDATELLI, J.S.; DALTOÉ, T.; ROSADO, J.O.; SPADA, P.; FORMOLO, F. Perfil do câncer de mama e relação entre fatores de risco e estadiamento clínico em hospital do sul do Brasil. Revista Brasileira de Oncologia Clínica. Vol.10. junho,2014. 
FELIX, L.M.C.; AOKI, T.; LIMA, S.M.R.R. Tratamento das ondas de calor em mulheres com câncer de mama. FEMINA, Maio 2010. vol 38 n $^{\circ} 5$.

Guide to cancer early diagnosis. Geneva: World Health Organization; 2017.

HEPPLER, C.D.; STRAND, L.M. Opportunities and responsibilities in pharmaceutical care. American Journal Hospital Pharmacy. Vol.47. Março de 1990.

HERNANDEZ, D.S., CASTRO, M.M.S., DÁDER, M.J.F. Método Dáder. Manual de Seguimento Farmacoterapêutico, 2009.

HICKEY M, SAUNDERS CM, STUCKEY BG. Management of menopausal symptoms in patients with breast cancer: an evidence-based approach. Lancet Oncol. 2005;6(9):687-95.

INSTITUTO NACIONAL DE CÂNCER JOSÉ ALENCAR GOMES DA SILVA. ABC do câncer: abordagens básicas para o controle do câncer / Instituto Nacional de Câncer. - Rio de Janeiro : Inca, 2011. 128 p. : il. ISBN 978-85.

- Sintomas do câncer de mama. Disponível: <http://www2.inca.gov.br/wps/wcm/connect/tiposdecancer/site/home/mama/sintomas > Acesso em: dezembro de 2017.

INUMANU, L.E. SILVEIRA, E.A. NAVES, M.M.V. Fatores de risco e de proteção para câncer de mama: uma revisão sistemática. Caderno de Saúde Pública, Rio de Janeiro, 27(7):1259-1270, jul, 2011.

IVAMA, Adriana Mitsue Ivama et al. Consenso brasileiro de atenção farmacêutica: proposta. Brasília: Organização Pan-Americana da Saúde, 2002. 24 p.

LAGARES, E.B.; SANTOS, K.F.; MENDES, R.C; MOREIRA, F.A.; ANASTÁCIO, L.R. Excesso de Peso em Mulheres com Diagnóstico de Câncer de Mama em Hormonioterapia com Tamoxifeno Revista Brasileira de Cancerologia 2013; 59(2): 201-210.

LANNIN, DR. Influence of socioeconomic and cultural factors on radical differences in late-stage of breast cancer. JAMA, 1998.

LEITE, F.M.C.; AMORIM, M.H.C.; CASTRO, D.S.; PRIMO, C.C. Mulheres com diagnóstico de câncer de mama em tratamento com Tamxifeno: perfil sociodemográfico e clínico. Revista Brasileira de Cancerologia, 2011.

LIVINALLI, A., FONSECA, C. L.; COUTO, D.H.N.; BORELLA, M.L.L.; TORRIANI, M. S. FALCARI, A. O. Manual de farmácia oncológica: quimioterápicos orais. $2^{\text {a }}$ edição. São Paulo, PlanMark,2016.

OLIVEIRA, R.S., MENEZES J.T.L., GONÇALVES, M.G.L. Adesão à Terapia Hormonal Adjuvante Oral em Pacientes com Câncer de Mama. Revista Brasileira de Cancerologia 2012; 58(4): 593-601.

Revista ENIAC Pesquisa, Guarulhos (SP), V.7, n.1, jan.- jun. 2018. 
PARTRIDGE, H.A., AVORN, J. WANG, P.S., WINER E.E. Adherence to Therapy With Oral Antineoplastic Agents. Journal of the National Cancer Institute, Vol. 94, No. 9, May 1, 2002.

RÊGO, IL.K.P.; NERY, I.S. Acesso e adesão ao tratamento de mulheres com câncer de mama assistidas em um hospital de oncologia. Revista de Cancerologia, 2013.

SOUZA, B.F.,MORAES, J.A., INOCENTI, A., SANTOS, M.A., SILVA, A.E.B.C. MIASSO, A.I. Mulheres com câncer de mama em uso de quimioterápicos: sintomas depressivos e adesão ao tratamento. Revista Latino-Americana de Enfermagem. Artigo Original set.-out. 2014;22(5):866-73.

STEWART B. W. and KLEIHUES P. (Eds): World Cancer Report. IARC Press.Lyon 2003.

STEWART B.W. and WILD, C.P. World Cancer Report. IARC Press. Lyon 2014.

TOKDEMIR G., KAV, S. The effect of structured education to patients receiving oral agents for cancer treatment on medication adherence and self-efficacy. Asia Pacific Journal Oncology Nursing. 2017 Oct-Dec; 4(4): 290-298.

VELOSO, R.S.; MANAÇAS, L.R.A.; SOARES, F.C.; FIGUEIRA, P.H.M. Análise da adesão à terapia antineoplásica oral de pacientes atendidos na farmácia ambulatorial do hospital do câncer II do Instituto Nacional de Câncer José Alencar Gomes da Silva. INCA, 2012.

Revista ENIAC Pesquisa, Guarulhos (SP), V.7, n.1, jan.- jun. 2018. 